\title{
Are We Ready For The Paradigm Shift In The Health Care System Of Pakistan?
}

\author{
Inayat H. Thaver, Nadia Khalid
}

Pakistan's health care indicators and accomplishment of its national as well international goals/targets though improving but are lagging the expected achievements ${ }^{1}$. The country is at a crossroads of having the demographic as well the epidemiologic transitions; besides these, social as well as mental issues are also increasing ${ }^{2}$. There is also a challenge of urbanization ${ }^{3}$ with its consequent flourishing of squatter settlements adding to burden on the health system.

Our health care systems have been traditionally focused on rural areas and catering for mother and child health, including the communicable diseases (especially in children) as they had been the main causes of mortality and morbidity ${ }^{4}$. Therefore, both curative and preventive (including promotive) care in the form of various levels of care from primary to tertiary and the famous Primary Health Care (in the aftermath of Alama Ata Declaration) have targeted mainly Communicable Diseases and some of the NonCommunicable Diseases (NCD) ${ }^{5}$. The selective NCDs that were considered such as cardio-vascular and metabolic disorders are mainly being treated and not prevented.

Similarly, demographically, we have moved on to 'population bulge' with a maximum proportion of adolescents and increasing trend of longer survival having older people ${ }^{6}$. Both these groups (adolescents and old) have got their emotional, social, reproductive and health requirements. The situation is further aggravated by the mushrooming but unregulated private (for-profit sector); perverse healthseeking behaviors, indiscriminate use of antibiotics and selfmedication. The old-age health delivery systems, stereotype (and focused on selective health issues) training institutions (and trainers) and lop-sided human resource production are not even mentally ready to think about these new emerging challenges. Besides, our EPI (Expanded Program for Immunization) and the vertical programs such as TB, Malaria, HIV/AIDS and others are yet to be controlled; the Polio eradication efforts are still not satisfactory ${ }^{7}$. We are also encountering epidemics of Dengue, HIV/AIDs, and Congo Fever etc. The geographical spread of these as well NCDs such as Diabetes, Hypertension, Cardio-vascular Diseases

Inayat Thaver
Professor, Head of Community Health Sciences
Bahria University Medical and Dental College, Karachi
Nadia Khalid
Sr. Lecturer Department of Community Health Sciences
Bahria University Medical and Dental College, Karachi
Received: 23-07-2019
Accepted: 01-10-2019

and mental/psychological problems are alarmingly increase with its health and financial consequences in terms of both treatment and rehabilitation and productive years lost.

The current malnutrition status, illustrates a combination of both undernutrition as well as the over nutrition or obesity ${ }^{8}$. Both male and female and even adolescent besides small children are victims of this status. Thus according to Epidemiological Transition, we are in the third phase of transition, wherein we are faced with the challenges of both communicable as well as non-communicable diseases along with old age and the big group of adolescent and malnourished population.

Pakistan's current government health care service delivery system is mostly focused for rural areas, with the probable exception of having the 'tertiary hospitals' located in larger cities $^{9}$. Thus the $40 \%+$ population residing in urban areas (half of which are in squatter settlements) have the main access to the tertiary hospitals; this is leading to the overcrowding and taking extra load of hospitals for just primary level health care facilities. Since there are inadequate outreach facilities in urban areas (especially in squatter areas), people are more used to seeking care by the nearby General Practitioners (GPs) or quacks, adding to their outof-pocket expenditure ${ }^{10}$. Let us not forget the emerging trends in morbidity \& mortality due to trauma and injuries including various disasters which are posing another burden to our ailing health system ${ }^{11}$.

Pakistan has original data sources in health, through various surveys such as Multiple Indicator Cluster Survey (MICS), Pakistan Demographic Health Survey (PDHS), National Nutrition Survey (NNS), Survey of Bureau of Statistics and other government supported institutions. We also have documented health policy and strategic approaches, however until now all of these have not been translated into implementation plans and improved health systems to address these emerging problems. The paradigm shifts are bound to happen, and we can already see the indicators related to high population growth, malnutrition, rapid urbanization, uncontrolled communicable and increasing proportions of non-communicable diseases all being addressed by same old system having human resource who emphasize more on curative care $^{12}$. The usual approach of having another "vertical" program is not going to make a big difference, as there is a growing feeling of having integration of programs; one example has been the IRMNCH (Integrated Reproductive Maternal \& Neonatal Health) Program. Unfortunately, there is no organized program for addressing the urban poor living in sums; the attention to over nutrition is the least; the old- 
age people are being neglected; common psychiatric problems (such as anxiety, depression) are treated as having somatic symptoms related to physical problems and for noncommunicable diseases, we are building the hospitals or specialized/state-of-art departments in hospitals.

How long should we be treating these above diseases and their consequences by neglecting the basic premise that, all of them, with few exceptions, can fairly well be prevented at an early stage; and healthy life style approaches can be easily promoted and implemented. We cannot take an ostrich approach and either ignore or wait for a miracle to happen through some international donor. We have to bring order to our own house. Each day wasted is accumulating more and more problems with consequent disastrous health and development outcomes. Considering the current sociopolitical, economic and security situations, the paradigm shift by the government may not be forthcoming though the WHO guidelines, recommendations and approaches are available. This may be because, "True paradigm shifts represent drastic, sometimes uncomfortable change. It is not surprising, therefore, that these events can be met with resistance as organizational leaders step outside their comfort zones".

We have traditionally been waiting for the government to take some action; let us also not forget that "Health is also each one of us' responsibility". A large difference can be made, If the medical, health and paramedic fraternity can make just an extra effort to spend some time to talk about (health education) how to prevent a disease that the patient has presented; and also encourage him/her for maintaining a healthy life style. Inspired by the quote of Gabriela Mistral I wish to extend it to all the population, by saying "To them, we cannot answer 'tomorrow', their (people) name is today".

\section{REFERENCES:}

1. Ali, S., Farooq, F., \& Chaudhry, I. S. (2012). Human Capital Formation and Economic Growth in Pakistan. Pakistan Journal of Social Sciences, 32(1), 229-240.

2. World Health Organization. Country Cooperation Strategy for WHO and Pakistan 2011-2017. Eastern Mediterranean. Regional Office. Cairo: 2013

3. Kugel man, M. 2014. Urbanization in Pakistan: Causes and Consequences. NOREF Expert Analysis

4. Health system profile of Pakistan. Regional Health Systems Observatory- WHO, 2007

5. Mortality Collaborators GBD Global, regional, and national under-5 mortality, adult mortality, age-specific mortality, and life expectancy, 1970-2016: a systematic analysis for the Global Burden of Disease Study 2016. Lancet. 2017; 390:1084-150

6. Durr e Nayab. Demographic dividend or Demographic threat in Pakistan. Pakistan Institute of development Economics, working Papers 2006:10

7. Hussain SF, Boyle P, Patel P. Eradicating polio in Pakistan: an analysis of the challenges and solutions to this security and health issue. Global Health. doi: 10.1186/s12992-0160195-3

8. National Nutrition Survey 2018. UNICEF Pakistan

9. Zahid G, Ali N, Bhutta ZA. Health Systems in Pakistan. In: Bhutta ZA, editor. Reproductive, Maternal, Child Health and Nutrition in Pakistan: opportunities for change. Karachi: Paramount Publishing Enterprise; 2013. p. 1-17

10. Sheikh, B. T., Rabbani, F., Safi, N., \& Dawar, Z. (2010). Contracting of primary health care services in Pakistan: Is upscaling a pragmatic thinking? The journal of the Pakistan Medical Association. 2010;60(5): 387.

11. Jennifer R. Davis, , Wilson S, Brock-Martin A, Glover S, and Erik R. The Impact of Disasters on Populations With Health and Health Care Disparities. Disaster Med Public Health Prep. 2010; 4(1): 30-38.

12. Islam S, Purnat TD, Phuong NTA. Non-Communicable Diseases (NCDs) in developing countries: a symposium report. Globalization and Health 2014; 10(81): 1-7 